UDC 1/14:530.145

Li ZHANG,

Lan $Z H A N G$

\title{
BIDIRECTIONAL INTERACTION: PREREQUISITE KNOWLEDGE AND QUANTUM THEORY
}

\begin{abstract}
The generation of any kind of theory cannot be separated from its specific social background, and it will certainly be marked by the era. Social factors restrict the existence and development of scientific theory and these factors do not directly affect scientific theory. Their influence on the production and development of scientific theory is realized through the intermediary of prerequisite knowledge. This paper aims to analyze the two-way interaction between knowledge of premises and quantum theory in the process of development of quantum theory from "classical" to "semi-classical" to "Non-Classical". Therefore, it points out how to break the shackles of the original "premise knowledge" in a timely and conscious manner, and how to construct its exploration of scientific work with the most appropriate background knowledge.
\end{abstract}

Keywords: premise knowledge, quantum mechanics, scientific dynamics, bidirectional Interaction.

The essence and core of the study of philosophy of science is scientific dynamics, and its process takes place and is constrained by the specific sociocultural context. In any reasonable sense, the premise knowledge is the background of a preset belief. Although scholars have different interpretations of premise knowledge, from the perspective of epistemology, scholars are basically consistent with the essence of premise knowledge.

\section{The Analysis of Premise Knowledge and its Structure}

The core of the premise knowledge structure is the world outlook, and the scientific revolution is essentially the transformation or change of world outlook.

\subsection{Ontological Level}

Under the Marxist scientific dynamics program, the major changes in the history of science are also regarded as fundamental changes in the view of nature. Proceeding from historical materialism, it must be the society that decides the change of world outlook.

After the industrial revolution, the development of productivity requires understanding the microscopic structure of matter. Understanding the microscopic structure of atoms exposied people to a completely new world, shaking the emotional foundation of the traditional world picture, and forcing people to look 
at the objective world in a new way. The mechanical view of nature since Newton has been broken. Absolute space-time, indivisible material minimum units, constant quality, Laplacian mechanical determinism have all been fatally challenged. Relative, hierarchical, and statistical concepts have become the basis of the new concept of nature.

\subsection{Epistemological Level}

The epistemology of the new era tends to be based on rationalism and is supplemented by intuitive epiphany. On the one hand, the construction and experimental design of the hypothesis are guided by a clear concept; On the other hand, this idea is closely related to the researcher's intuitive choice of natural ontological picture.

For such cutting-edge scientific research cases as quantum theory, we need to pay attention to the following issues:

First, knowledge of premises is a "mixture".

On the one hand, it is not a so-called "theory" in terms of the internal structure and composition of premise knowledge. Because, in a large number of scientific experimental cases, these premise knowledge can hardly be consistent with each other; On the other hand, knowledge of premises is not the scientific whole that has been provided. As a background of presupposition belief, premise knowledge plays a specific and limited role in a series of assumptions, the implementation of scientific activities, and scientific interpretation of scientific research.

Second, knowledge of premises is by no means "anything". Admittedly, there is often room for debate about the relevance of certain background elements, but "the background be- lief is by no means loaded with some kind of arbitrary theme," and it is definitely not "anything at all". On the contrary, in most cases of scientific background, there is a lack of choice and few alternatives.

\subsection{Axiology Level}

The scientific community of society is always in a certain socio-economic and political relationship, and the values of society inevitably limit the perspective of these communities and their members.

In the summer of 1918, German physicists, like the rest of the German public, still confidently expected Germany to win the war, and the public praised their achievements. Thanks to Germany's advanced industrial technology and the economic and military power that industry guarantees, their value is supreme and even arrogant in the public eye. However, at the end of 1918, Germany's completely unexpected military failure and industrial collapse brought about a major change in public attitudes and even a campaign against industrial idols.

Scientists find themselves facing a dramatic scale of public value. With the end of the war and the arrival of peace orders, the belief of the rational world has been shaken, and people are looking for ways to help themselves in an irrational world order. Endorsement of irrationality and mysticism has become the main ideological feature after the war. This new trend of thought is mainly emphasizing living bodies rather than machinery. The concept of value, goal, and purpose replaces the mechanical causality law. In this way, a general sense of crisis has formed in the German academic community. This is a crisis of morality and culture, a crisis of science and learning. Human free will, 
self-desire, and psychological desire have become the primary starting point. This kind of value orientation inevitably leads to the rejection of mechanical determinism and metaphysical causality. In short, these external influences lead physicists naturally to "non-causal" quantum mechanics.

\section{The Development of Quantum}

Theory and its Relationship with

Prerequisite Knowledge

We can roughly divide the establishment process of quantum theory into three stages: The first stage is the proposal of quantum concepts and their application in solving certain specific problems, approximately from Planck proposing quantum concepts to Bohr establishing an atomic model; In the second stage, on the basis of classical theory, quantum concepts are introduced, microscopic physical models are constructed, and on the basis of this model, semi-classical theories are established. This process is approximately from Bohr's proposed atomic model to De Broglie's proposed material wave concept. Before, in the third stage, quantum mechanics was established and perfected in mathematical form and physical interpretation, from about 1923 to 1927 . We can call the first stage "classic stage", the second stage as semi-classical stage, and the third stage as "Non-Classical stage".

\subsection{Classic Stage}

The development of physics has roughly gone through three major stages. That is, ancient physics, classical physics and modern physics. From one stage to another, major revolutionary changes have taken place. The basic characteristics of ancient physics are intuition and speculation. The basic form is a description of phenomena and empirical nature. It is basically subordinate to speculative natural philosophy. Classical physics has three pillars, namely classical mechanics, classical thermodynamics (including statistical physics) and classical electromagnetic theory. The classical mechanics - laid down by Galileo and Newton - began with the negation of Aristotle's mechanical point of view. It is the first mature scientific theory system established by modern natural sciences. It is also the general theoretical concept and scientific method concept of modern natural sciences. Classical thermodynamics and statistical physics introduced the concept of irreversibility and statistical concepts into physics, while classical electromagnetic theory further aroused the concept of field. However, their theoretical basis is still the general concept of classical mechanics. Therefore, when analyzing the basic assumptions and basic characteristics of classical physics, we naturally take classical mechanics as a sample.

\section{First, the Basic Characteristics of Classical Mechanics}

We can summarize the basic features contained in classical mechanics as follows: the simple assumption of the world; The invariable assumption of the nature of matter; Mechanic characteristics; The universal assumption of the law of mechanics; Basic law deterministic characteristics; Decisive assumptions of time and space; The separation and intuitive reflection of human and nature; The cognitive methods are mainly analytical and experiential; The absolute objectivity of scientific theory. These 
basic assumptions of classical mechanics reflect the level of development and general concepts of scientific understanding in that era, and basically represent the basic characteristics of the entire classical physics. This basic feature has a profound influence on the development of modern physics (only quantum theory is discussed here).

Second, Planck Proposed the "Energy Quantum Hypothesis"

The "quantum concept" was the beginning of the scientific revolution in the last century. However, Planck himself was not a conscious revolutionary. When he began this work, he did not intend to break through the classical theory. Even after the quantum concept was proposed for a long time, Planck himself did not fully realize the significance of this discovery, but only regarded the quantum concept as a computational expediency.

Planck's discovery process can be divided into two stages: Before October 19, 1900, Planck first defined the electromagnetic entropy on the basis of thermodynamics and electromagnetism, and deduced the consistent results with W. Wien's, but when he found that the Wien formula did not meet the experimental results, he took the two limit formulas lamb $\mathrm{T} \rightarrow 0$ and lamb $\mathrm{T} \rightarrow$. In order to eliminate the emission results, the mathematical interpolation method is used to find a new formula that is exactly in line with the experiment.

The new formula introduced by Planck seems to indicate that the oscillator can only contain discrete energy Quanta; but this result is so different from anything known in classical physics, Planck found it incredible because of the deep-rooted classical view of nature. The only way out is to assume that when the material absorbs and emits radiation, the energy does not continuously change, but jumps at an integer multiple of a certain value. In the jump change, this inseparable minimum energy unit is called an energy quantum, with a value of $h \gamma 0$ ( $\gamma 0$ is the frequency of the oscillator; $\mathrm{H}$, as a quantum of action, is a universal constant).

This is the quantum hypothesis. It broke the long-held concept of "nature without jumping" and proclaimed the birth of quantum theory. The growth of the quantum hypothesis was not smooth, and the physics community responded coldly. They only recognized the Planck formula but opposed the Planck hypothesis. J. W. S. Rayleigh, H. A. Lorentz and other famous physicists at the time almost did not admit it. Even Planck himself felt that he was too bold.

Planck did not intend to break the classical theory, but only used the concept of "quantum" as a computational expediency. He believes that this is a helpless action that has been forced by the facts. In fact, he spent more than ten years in vain trying to pull his hypothesis back to the track of classical theory. The spark of quantum theory, although fortunately ignited by Planck, was almost extinguished by his own hands.

Third, the Mutual Mapping of Quantum Concepts and German Reality

Why did Planck regress after he proposed the epoch-making concept of energy quantum?

The deep reason lies in the fact that the quantum world and the real world in Weimar period constitute a kind of symbiotic mutual 
appearance, or a mirror metaphorical relationship. According to classical physics, energy radiation is continuous and it propagates like a water wave. Therefore, Planck can only think that matter must absorb or release energy one by one; Outside of matter, energy propagation still conforms to Maxwell's electromagnetic theory, and it also travels continuously like a water wave (see Maxwell, 1855). This is essentially a compromise to classical theory. It is an incomplete quantum theory. And this compromise is actually a remnant of authority.

It can be said that Planck's radiation law is still a semi-empirical law. Only according to the Wien formula and the Rayley-Kings formula, according to the empirical curve of the experiment, the interpolation method "comes together". Since Planck has long been influenced by classical physics in concept, and is convinced of classical physics, he always uses classical physics as the standard in an attempt to incorporate quantum $\mathrm{H}$ into classical physics. It can be seen that although a new concept has emerged under the impetus of facts, it is only possible to break the shackles of premise knowledge and break through traditional ideas and grow up. It is only possible through the scientific ideological revolution ("paradigm shift").

\subsection{Semi-Classic Stage}

The first to apply quantum concepts to other problems was Einstein. His theory of light quantum successfully explained the phenomenon of photoelectric effects and brought the ancient problem of "the struggle of the nature of light" into a new stage, that is, the theory of "wave-particle duality of light". It gives people a further understanding of the nature of light: light sometimes shows volatility, sometimes shows particle nature, and it is neither a classical particle nor a classical wave. It was Einstein's work that enabled quantum theory to develop in the first ten years.

In the summer of 1912, Boergrass wrote a memorandum discussing the structure of atoms and molecules, setting the electrons in the atom to move on the ring around the core, but he felt that classical mechanics could not determine the size of the ring and the cycle of electronic motion. In 1913 Bohr wrote the classic atomic structure paper. The main contribution of this paper is to propose quantization conditions and frequency formulas. And his principle of correspondence had a major influence on the development of later quantum theory. It is believed that the various studies that led to quantum mechanics between 1919 and 1925 can be regarded as a series of guesses guided by the corresponding principles.

\subsection{Non-Classical Stage}

First, the Material Wave of De Broglie

Bohr's theory is based on the combination of classical mechanics of electronic motion and quantum conditions that are imposed on classical motion only in order to define the discrete States of the system. De Broglie started from Einstein's special theory of relativity. After thinking of particles as waves, he naturally explained the quantization conditions in the Bohr atomic model with the concept of standing waves that can be understood by classical theory. Each fixed state of the atom is equivalent to a kind of Debuluoyibo. In this way, the contradictions within Bohr's theory are eliminated. As a result, quantum conditions are associated with 
wave-particle duality, and quantum conditions have always been an external factor in classical electrodynamics and are now an intrinsic and inevitable attribute. The concept of material waves pointed out by De Broglie was not based on any experimental basis at that time. It was completely derived from existing theories and rigorous and concise mathematical and logical reasoning. This is one of the main features of the new quantum theory, the scientific discovery of the quantum mechanics phase, which is different from the old quantum theory.

\section{Second, Schrodinger Equation}

E. Schrodinger was inspired by De Broglie's original ideas. De Broglie describes microscopic particles with material waves, but has not yet used a wave function to establish a wave equation that represents the movement of microscopic particles. Therefore, Schrodinger tried to use the wave function to establish such an equation and make the quantization conditions in Bohr's theory a natural result of the solution of this equation. He started from the de Broglie formula, used W. R. Hamilton's classic wave equation and finally came to a wave equation reflecting the law of motion of three-dimensional free particles in 1926, the famous Schrodinger equation. The position of Schrodinger's equation in quantum mechanics, like Niudunfangcheng in classical mechanics and Maxwell's equation in classical electrodynamics reflects the basic laws of the movement of microscopic objects (see Maxwell, 1855). Before Schrodinger established the wave equation, Heisenberg set aside the concept of "orbit" in classical physics, started with considerable measurements, and established matrix mechanics with the help of Born and Jordan (see Heisenberg, 1985). Later, after Dirac's improvement, it became a theoretical system with complete concept and logical consistency. This is a further departure from the classical theory, and it is also the most complete deviation. In March 1926, Schrodinger discovered that these two theories are mathematically equivalent. Since then, the two major theories have been collectively referred to as quantum mechanics.

\section{Conclusion}

From the development of "classic" "semi-classic" - "non-classic", we can see that before the quantum theory was proposed, people were in contact with the daily world. Various rational formalistic and sacred ideas about the "mysterious" world have been produced. It contains some vague intermediary but successful pre-control method. With the transformation of these driving methods and gradually becoming an exploratory method, the scientific tradition (quantum theory) that has been promoted and developed by these driving methods will emerge in the theory of competition and become a winner. However, this kind of premise knowledge is not completely determined, nor is it necessarily moving toward irreconcilability and relativism. Instead, it needs to construct its interpretation of scientific exploration work with the most appropriate background knowledge.

In summary, it is not difficult to find that "premise knowledge" is often expressed in a potential way as some normative binding principles, forming a thinking trend. Therefore, it is important and valuable how to break the shackles of the original "premise knowledge" in a timely and conscious manner and become a self-conscious revolutionary. 


\section{REFERENCES}

Forman, P. (1971). Weimar Culture, Causality, and Quantu Theory, 1918-1927. Historical Studies in the Physic Science, 3(25), 1-115.

Guy, P. (2005). Weimar Culture: a Short and Bright Cultural Legend. (L. Senyao Trans.). Hefei: Anhui Education Press.

Heisenberg, W. (1985). Development and Society of Atomic Physics. China Social Sciences Press.

Kraft, P., \& Krose, P. (1984). Adaption of Scientific Knowledge to an Intellectual Environment. Paul Forman's 'Weimar Culture, Causality, and Quantum Theory, 1918-1927'. Analysis and Criticism. Centaurus, 27(1), 76-99.

Kuhn, Th. S. (2003). The Structure of the Scientific Revolution. Beijing: Peking University Press.

Maxwell, J. C. (1855). On Faraday's Lines of Force. In W. Niven (Ed.), The Scientific Papers of Jame Clerk Maxwell (pp. 155-229). Paris: Librairie Scien- tifique J. Hermann.

Mutian, S. (1996). Outline of the Third Kind of Knowledge. Dialectics of Nature Communication, № 1.

Mutian, S. (2007). On the Two Convergence of Scientific Dynamics - a Comparison between Western and Russian (Soviet) Philosophy of Science. Dialectics of Nature Communication.

Pinson, K. S. (1987). Modern German History: Its History and Culture (Vol. II). (V. Deryi, Trans.) Beijing: Comercial Press.

Stepin, V. S. (1987). Nauchnye revolyutsii kak «tochki» bifurkatsii v razvitii znaniya (Scientific Revolutions as "Points" of Bifurcation in the Development of Knowledge). In V. S. Stepin (Ed.), Nauchnye revolyutsii $v$ dinamike kul'tury (Scientific Revolutions in the Dynamics of Culture, in Russian). Minsk.

Suppe, F. (Ed.). (1977). The Structure of Scientific Theories. Urbana: University of Illinois Press. 